\title{
Inclusión de Estudiantes Sordos/as en Escuelas Regulares en Chile: Posibilidades y Limitaciones desde un Análisis de Prácticas de Aula
}

\section{Deaf Students' Inclusion in Chilean Regular Schools: Possibilities and Limitations from an Analysis on Classroom Practices}

\author{
Sofia Larrazabal * \\ Rosario Palacios \\ Victoria Espinoza \\ Pontificia Universidad Católica de Chile, Chile
}

\begin{abstract}
La implementación de políticas educativas inclusivas en Chile ha sido un proceso que no ha estado exento de dificultades. Este artículo explora diferentes prácticas sociales e institucionales desarrolladas durante la escolarización de estudiantes sordos/as. Se analizan interacciones entre estudiantes, docentes e intérpretes en clases con estudiantes sordos/as, interacciones sociales entre estudiantes, disposiciones escolares respecto a la inclusión y, por último, aspectos administrativos y financieros relacionados al modelo de política pública imperante. A través de una aproximación a dos escuelas públicas en Santiago de Chile, profundizamos en los modos en que el estudiantado sordo se desenvuelve entre la integración y la inclusión. Basándonos en observaciones de clases y entrevistas con profesores, intérpretes, estudiantes y directivos de comunidades escolares, describimos hallazgos respecto de la significación de la lengua de señas, los roles de intérpretes y educadores, el aprendizaje comunitario de la lengua de señas y el rol del aula de recursos. Se discuten los resultados en torno a las condiciones necesarias para la inclusión de estudiantes sordos, describiendo las prácticas docentes como un elemento clave para la generación de una verdadera inclusión dentro del aula, y las prácticas culturales de la comunidad escolar como posibles favorecedoras de la inclusión fuera de ella.
\end{abstract}

Descriptores: Estudiantes sordos/as; Integración escolar; Inclusión; Metodología cualitativa; Lengua de señas.

The implementation of inclusive education policies in Chile has been a process that has not been without difficulties. This paper explores numerous social and institutional practices developed during the schooling process of deaf students. We analyze interactions between deaf students and their peers, teachers and interpreters during classes, institutional dispositions regarding inclusion, and administrative and financial issues related to mainstream educational public policy. Through an approach into two public schools in Santiago de Chile, we delve into the ways in which students deal between integration and inclusion. Based on class observations and interviews with students, teachers, interpreters and school principals, we describe how Chilean legal framework allows to integrate deaf students into regular classrooms. The findings are analyzed regarding the significance of sign language, the academic roles that sign language interpreters must maintain, the tensions regarding the integration of deaf students, the need of learning sign language by the entire educational community, and the use of classroom resources in the education of deaf students. The results are discussed regarding the necessary conditions for deaf students' inclusion, describing teaching practices as a critical element for achieving true inclusion within the classroom, and school community's cultural practices as favoring inclusion outside it.

Keywords: Deaf students; School integration; Inclusion; Qualitative methodology; Sign language.

*Contacto: sofia.larrazabal@gmail.com

ISSN: 0718-7378

www.rinace.net/rlei/
Recibido: 14/07/2020

$1^{\text {a }}$ Evaluación: 25/01/2021

Aceptado: 28/04/2021 


\section{Introducción}

Chile ha avanzado en modificar sus leyes apuntando hacia un modelo más inclusivo ante la diversidad cognitiva y funcional, adoptando leyes y decretos sobre: inclusión de estudiantes con necesidades educativas especiales (NEE) en escuelas regulares (Ley $\mathrm{n}^{\circ}$ 20.845/2005); subvenciones adicionales para estudiantes con NEE (Ley $\left.\mathrm{n}^{\circ} 20.201 / 2007\right)$; especificaciones diagnósticas y administrativas relativas a dicha inclusión (Decreto Supremo $\left.{ }^{\circ} 170 / 2009\right)$; normas sobre igualdad de oportunidades para personas con discapacidad (Ley $\mathrm{n}^{\circ} 20.422 / 2010$ ); y criterios y orientaciones de adecuación curricular para estudiantes con NEE en Educación Parvularia y Educación Básica (Decreto Exento nº83/2015) (García y López, 2019; Herrera y de la Paz, 2019). En relación a la sordera específicamente, la literatura indica un correlato en materia de derecho internacional en el reconocimiento del derecho de las personas sordas a recibir una educación bilingüe (Congreso de Hamburgo) y un trato justo e inclusivo (Convención de Derechos de las Personas con Discapacidad, ratificada por Chile en 2008).

Sin embargo, este grupo de regulaciones ha sido criticado por diferentes actores dentro del sistema educativo: estudiantes, docentes, directivos escolares y padres, entre otros. Se ha planteado que la normativa apela más bien a la integración que a la inclusión de niñas/os con NEE, debido a que el cuerpo docente responde a un cuerpo legal amplio caracterizado por la poca sistematización, el foco en la rendición de cuentas y la falta de criterios comunes que faciliten la interpretación de las normas. Los/as docentes y las comunidades escolares perciben una extrema laxitud en las definiciones que entregan los decretos sobre qué es la inclusión y cómo se lleva a la práctica en las salas de clases, pues en un contexto en que el número de estudiantes por atender y educar en cada sala de clase es alrededor de 40, se apunta hacia cumplir con criterios numéricos, usualmente asociados a la mantención del número de matrícula por asuntos financieros como los voucher, o a números mínimos y máximos de alumnos/as con NEE permitidos por sala- (Fardella et al., 2016; Sisto, 2019; Villalobos y Quaresma, 2015), más que hacia desarrollar espacios de diseño y planificación de clases y climas de convivencia escolar que permitan acoger de manera equilibrada las capacidades y necesidades educativas de todos/as los/as estudiantes (Canals et al., 2019). Como consecuencia, los actores involucrados interpretan y ponen en práctica la ley estableciendo prioridades que no siempre están acorde a sus voluntades profesionales o personales (Palacios et al., 2019).

Por tanto, no hay un único modo ni contexto para organizar una respuesta educativa ajustada a las necesidades del alumnado sordo (Huaiquinao y Rebolledo, 2019). Sin embargo, hay consenso sobre la necesidad planificar cuidadosamente en función de características territoriales, del número de alumnos con pérdidas auditivas, de los recursos disponibles, y de la capacidad de agencia de los actores educativos implicados en las escuelas (Domínguez, 2009; Palacios et al., 2019). A continuación presentamos hallazgos situados en la experiencia de estudiantes sordos/as en escuelas regulares, ilustrando el despliegue de algunas de las prácticas de aula recurrentes para su integración. Mediante ellos no pretendemos reducir ni representar tendencias en la escolarización de estudiantes sordos/as, sino más bien aportar a la comprensión de la diversidad desde la subjetividad de estudiantes, intérpretes de Lengua de Señas chilena (LSch) y profesores/as al momento de poner en práctica tanto mandatos como afectos. Concluimos que existen desafíos institucionales para la incorporación de elementos culturales que promuevan la inclusión en escuelas regulares (tales como la posibilidad de aprendizaje bilingüe), así como también 
desafíos para que profesores/as e intérpretes encuentren alicientes en la diversidad lingüística dentro del aula, en favor de la adaptación y la diversificación de los procesos de enseñanza y aprendizaje.

\section{Marco teórico}

Comenzar a comprender el funcionamiento de un grupo particular dentro de un contexto institucionalizado como la escuela, en este caso, la comunidad sorda en escuelas regulares, requiere establecer algunas características y límites. Aunque la sordera es un elemento compartido por muchos/as estudiantes que actualmente asisten a escuelas regulares, no todas las personas sordas se desenvuelven del mismo modo ni requieren los mismos recursos interpretativos para comunicarse (Swanwick, 2015). Sin embargo, los/as sordos/as comparten una experiencia de audición diferenciada a la de la norma oyente, lo que los/as hace parte no sólo de cualidades sino que también de asignaciones o pérdidas tanto prácticas como simbólicas relacionadas a la ausencia de un sentido. En particular a esta ausencia, por 'sordera' podemos abarcar:

Un grupo heterogéneo de variaciones en la capacidad de oír. Este término engloba
tanto a los sujetos con sordera, como a los que tienen hipoacusia, además incluye a
quienes presentan la deficiencia auditiva (DA) desde su nacimiento y a aquellos que
la adquirieron posterior al aprendizaje de la lengua oral. (Valles, 2003, p. 93)

$\mathrm{Al}$ 'englobar' a un diverso grupo de personas que presentan otras múltiples características personales, compartir la sordera genera lazos de pertenencia e identidad que devienen en una cultura ${ }^{1}$, materializada en normas, costumbres y creencias que, aunque fundamentales para el posicionamiento de cada persona dentro de su sociedad, son vistas de modo particular en función de las experiencias de pertenencia de cada quien (Bernal-Martínez, 2008; Huaiquinao y Rebolledo, 2019). Además, se desarrollan y adquieren mediante la relación con otros a través del lenguaje, el diálogo y la interacción con el entorno. Es el lenguaje lo que va "tejiendo una entramada red de significados que originan valores, creencias y mitos que dan lugar a las prácticas sociales de ese grupo particular" (Habermas, 1989, citado en Damm y Silva, 2017).

Un buen espacio para observar el desarrollo del lenguaje y los significados que porta son las escuelas donde los/as miembros de una comunidad lingüística sorda se desarrollan y conviven dentro de una comunidad lingüística oyente. Al respecto, abordaremos teóricamente la relación entre estudiantes sordos/as e instituciones escolares desde dos perspectivas: procesos de integración e inclusión a la escuela, y estudios académicos sobre modelos para la inclusión de personas con discapacidad y NEE. Ante el derecho a la educación de todas las niñas y niños sin importar sus habilidades o discapacidades, o las dificultades que presenten durante el aprendizaje (Warnock, 2005), la escolarización de los estudiantes sordos/as ha avanzado desde una perspectiva de integración clínicaterapéutica basada en los déficits, hacia una concepción socio-antropológica basada en capacidades e igualdad de oportunidades, de acceso y de participación (Domínguez, 2009; Mineduc, 2007; Ruiz, 2016). El enfoque socio-antropológico implica la construcción de

${ }^{1}$ Entendida aquí como numerosas asignaciones, actitudes, adquisiciones y pérdidas de símbolos y signos, además de múltiples cualidades que se transmiten y heredan históricamente para comunicar y/o perpetuar conocimientos (Geertz, 2003). 
sistemas educativos "inclusivos" (Damm y Silva, 2017), sin embargo, esto sólo puede ocurrir si las escuelas regulares son capaces de educar a todos/as en su comunidad, privilegiando la equidad más que la igualdad (Ruiz, 2016; Vesga y Vesga, 2015).

Por su parte, los estudios sobre modelos para la inclusión se caracterizan por un foco en la justicia, la superación de barreras y el respeto a la diferencia a través del acceso a derechos y a la igualdad de oportunidades (Ainscow, 2008; Ainscow et al., 2006; Domínguez, 2009; Echeita y Duk, 2008; Villalobos et al., 2015). En el debate educativo es posible observar posturas que ven la discapacidad como producto de factores individuales, y otras que la ven como producto de las limitaciones de los sistemas educativos para adaptarse a la diversidad de los/as niños/as (Terzi, 2005). En el caso de la literatura sobre educación y sordera, la tendencia ha sido a analizar el aprendizaje de los/as estudiantes a través de planos separados: aspectos audiológicos de la sordera y desarrollo del lenguaje por una parte, y por otra, políticas de inclusión y contextos socioculturales (Herrera y de la Paz, 2019; Huaiquinao y Rebolledo, 2019; Swanwick, 2015).

Sin embargo, afirmamos que estas separaciones y dualidades entre elementos individuales y sociales son oposiciones artificiales, pues estos planos pueden ser vistos en conjunto a través de un modelo ecológico en el que el aprendizaje se comprenda como un proceso que involucra dominios físicos, cognitivos, sociales y afectivos a lo largo de un ciclo vital, poniendo énfasis en que las personas ponen todo de sí incluyendo sus sentidos como un vehículo para comprender e interpretar el espacio que nos rodea, atendiendo a contextos e interacciones sociales, culturales, lingüísticas, identitarias, entre otras, que determinan la experiencia de comprender y aprehender un contenido (Knoors y Marschark, 2014; Swanwick, 2015; Terzi, 2005). Encontramos un correlato anterior a este modelo ecológico en el enfoque basado en las capacidades. Este enfoque, proveniente del trabajo sobre justicia y desigualdad de Amartya Sen (1992), propone que las interrelaciones entre factores individuales, sociales y circunstanciales son centrales para evaluar las capacidades de una persona, es decir, las oportunidades que realmente tiene de alcanzar y mantener funcionalidades (seres y quehaceres que los individuos sociales valoran). Así, se propone la valoración de la diferencia como un elemento constitutivo humano (en materias personales, circunstanciales y de habilidades) y por lo tanto, el diseño de conceptualizaciones y contextos que tomen en cuenta el carácter relacional y multifactorial de las limitaciones y las necesidades especiales. En resumen, se presume que los impedimentos individuales, en caso de afectar la funcionalidad, pueden convertirse en discapacidades (es decir, restricciones al logro del bienestar). Así, repensar la discapacidad desde este enfoque requiere considerar todo el abanico de capacidades a las que una persona puede acceder, y evaluar el impacto de sus impedimentos personales y contextuales en la libertad de elegir entre ese abanico (Sen, 1992; Terzi, 2005).

$\mathrm{Al}$ respecto, Lorella Terzi (2005) propone utilizar este enfoque en la resignificación de lo que se entiende como discapacidad y NEE, para la superación del dilema de la diferencia ${ }^{2}$ en la educación, permitiendo, entre otras cosas: equilibrar el peso de los factores individuales y de los factores sociales en la construcción de discursos sobre discapacidad

\footnotetext{
${ }^{2}$ La aparentemente inevitable situación de elegir entre identificar la diferencia en un/a niño/a para proveer atención especializada -a riesgo de etiquetar o separar-, o acentuar la semejanza y ofrecer una atención estandarizada a todos/as - a riesgo de no proveer lo que es relevante y necesario para niños en particular- (Terzi, 2005, p. 444).
} 
y NEE y poner el foco en que las capacidades son potenciales, diversas y pueden desarrollarse mediante el funcionamiento alternativo (es decir, hacer la misma actividad pero de otra manera).

Existe consenso sobre la necesidad de cambiar el modelo de integración, donde la persona sorda es quien se adapta a la comunidad mayoritaria oyente, sacrificando sus propias subjetividades personales y culturales, por un modelo de inclusión en el que los entornos social, escolar y familiar son los que se adaptan, desarrollando imaginarios colectivos con actitudes y prácticas positivas hacia la diversidad (Swanwick, 2015; Vesga y Vesga, 2015). Las experiencias señalan que lo importante es la capacidad de los sistemas educativos para encontrar soluciones adaptadas a las necesidades de estudiantes sordos/as, que permitan su desarrollo lingüístico, emocional, social y académico de manera integral (Antia et al., 2002; Domínguez, 2009; Giorcelli, 2004; Knoors y Marschark, 2014; Powers, 2002). Así, cuando la escuela reciba al alumnado sordo, deberá poner en marcha una serie de medidas educativas que contribuyan a eliminar barreras en la comunicación, la interacción social y el aprendizaje -organización de apoyos, elección del método de comunicación, uso de ayudas técnicas para la audición y la comunicación, intervención de profesionales especializados, adaptación del currículum, utilización de estrategias didácticas, etc.(Camacho y Pérez, 2014; Ruiz, 2016).

Considerando los datos teóricos anteriores y sobre la base de un marco legal que propende a la generación de políticas inclusivas, es interesante conocer la manera en que se ejecutan y cómo se han puesto en práctica las políticas inclusivas respecto del alumnado con DA. Según datos aportados por la Unidad de Educación Especial del Mineduc al año 2019, 369 estudiantes sordos/as asistieron a escuelas especiales y 1.842 estudiantes fueron integrados en escuelas regulares a través de Programas de Integración Escolar (PIE). La situación comunicacional de los estudiantes es muy distinta según su tipo de escolarización: mientras las/os estudiantes pertenecientes a escuelas especiales reciben su educación por medio de LSch el 100\% de su jornada escolar, los/as sordos/as integrados en PIE cuentan con intérprete de LSch sólo en parte de ésta. La diferencia en matrícula es similar a la diferencia en oferta: las escuelas especiales son en su mayoría para la enseñanza de estudiantes con trastornos del lenguaje, mientras que sólo 10 escuelas se dedican a la enseñanza de estudiantes con $\mathrm{DA}^{3}$ (Holz, 2018).

El desarrollo del lenguaje en las escuelas, y su ausencia, tiene consecuencias importantes para procesos de aprendizaje a mediano y largo plazo. Según los primeros resultados de un estudio de Catalina Santa Cruz et al. (2019) presentados en la Figura 1, los/as sordos/as en escuelas especiales muestran un mayor desarrollo en los precursores de lectura y razonamiento matemático que estudiantes de escuelas regulares. Esto podría indicar que sus aprendizajes se ven beneficiados por la posibilidad de aprehender el contenido a través de códigos lingüísticos propios, con dinámicas comunicacionales compartidas con profesores/as y compañeros/as, en vez de tener que traducir e interpretar las clases de manera particular en un ambiente aislado y dominado por dinámicas basadas en la oralidad y la escucha.

\footnotetext{
${ }^{3}$ Corregido a 2020 según datos aportados por la Unidad de Educación Especial del Mineduc.
} 


\section{Figura 1}

Comparación de puntajes obtenidos en la evaluación de precursores de lenguaje y matemática en estudiantes sordos/as de escuelas especiales y escuelas regulares

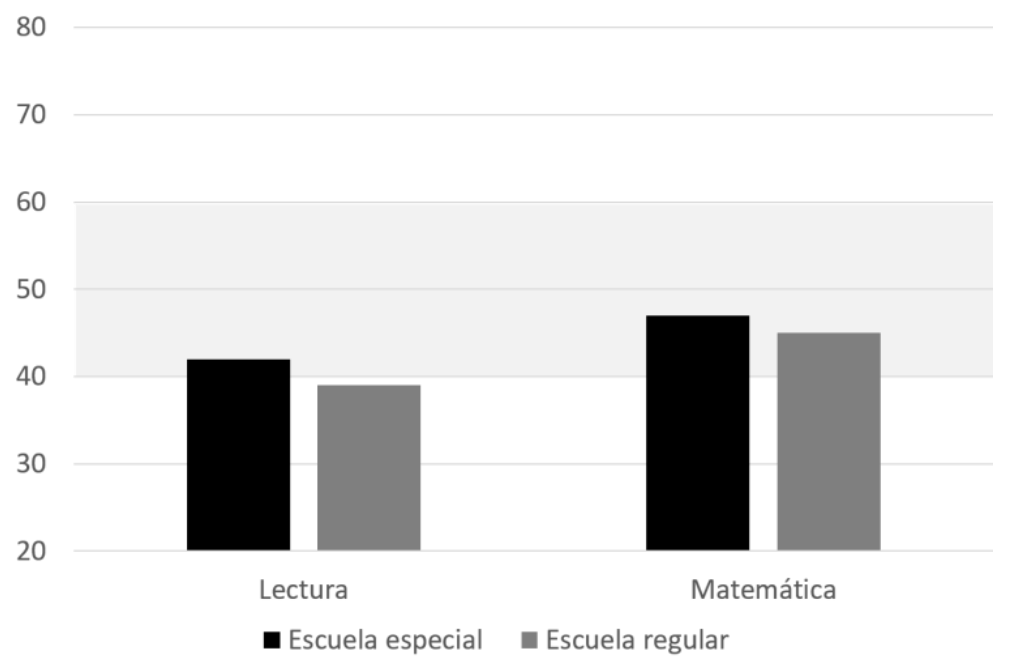

Nota. Elaboración propia en base a los datos aportados por los autores (Santa Cruz et al. 2019). Los puntajes se expresan en puntaje $\mathrm{t}$ (promedio 50, desviación estándar de 10).

Creemos que para abordar la forma en que se educan los estudiantes sordos en Chile es fundamental tener en cuenta la existencia de una cultura Sorda. De la Paz y Salamanca (2009) aportan cuatro elementos verificadores de la misma que son necesarios de tener en cuenta: 1. la dimensión simbólica dada por la existencia de la lengua de señas, 2. la presencia de lo emocional, dada por sentimientos tales como desconfianza, frustración, falta de comunicación o menos valorización provocados por la colonización lingüística, 3. la presencia de conocimientos, dada por la transmisión de la cultura, y 4. la dimensión material, dada por los objetos culturales como la LSch y las manos. Por otra parte, es central tener en cuenta cómo esa cultura se relaciona con la cultura oyente, para lo cual las aproximaciones desde la interculturalidad son necesarias de incorporar. Carolina Becerra $(2013,2020)$ destaca los siguientes elementos como básicos para la existencia de una interacción entre culturas: 1. alteridad, es decir, que exista reconocimiento de la cultura que no es la propia, y por ende, de la persona alterna como parte de una cultura diferente, 2. lengua, que significa que se debe buscar una forma de comunicación posible para ambas culturas, sin hacer de la lengua de una de las culturas la hegemónica, 3. intercambio, que apunta a que existan instancias de experiencias comunes entre ambas culturas lo que implica, a su vez, que no se considere lo propio de una cultura normal y lo de la otra anormal, y 4. reciprocidad de experiencias, que tiene que ver con las ganancias que ambos grupos obtienen de la interacción dialógica, sobre todo en cuanto a expandir horizontes y entender el mundo de una forma inclusiva.

Hasta la fecha, hay estudios que abordan la importancia de que estudiantes sordos aprendan la lectura y la escritura en un ambiente bilingüe, como pretende la escuela especial (Herrera y De la Paz, 2019; Lissi et al. 2012; Morales, 2019; Morales y Morales, 2018). Sin embargo, como hemos señalado, la realidad chilena muestra que la mayoría de los estudiantes sordos asisten a escuelas regulares. 


\section{Método}

Los relatos presentados en este artículo son extractos de un estudio mayor ${ }^{4}$ que tiene por objetivo describir y analizar políticas públicas orientadas a la inclusión educativa de estudiantes con NEE. Para tal estudio, se seleccionaron seis establecimientos educacionales en Santiago de Chile, tres gratuitos municipales o del Servicio Local de Educación, y tres particulares subvencionados con y sin copago. Las escuelas seleccionadas obedecen también al criterio de pertenecer a comunas de bajos ingresos en las que los/as estudiantes no cuentan con recursos adicionales para terapias o apoyos fuera de la institución, y a las cuales concurren estudiantes con diversas condiciones de discapacidad y/o necesidades educativas especiales, entre ellas, con DA. En este artículo, ahondamos en los contextos de dos escuelas (una mixta y una de niñas) en las que hay estudiantes con sordera o hipoacusia. Realizamos observaciones no participantes en dos asignaturas (lenguaje y matemáticas) impartidas por duplas docentes compuestas por profesores/as regulares y sus colegas de educación especial. En paralelo sostuvimos entrevistas semi-estructuradas con estudiantes, docentes de educación especial, intérpretes y directivas/os de las escuelas (Álvarez, 2008; Flores y Naranjo, 2013). La participación de todas las personas fue informada y voluntaria, además de asignarse pseudónimos a sus relatos. El registro de las observaciones se realizó mediante notas de campo y las entrevistas fueron grabadas en audio y transcritas.

Nuestros hallazgos dialogan con prácticas etnográficas y antropológicas registradas en estudios previos (Herrera y de la Paz, 2019; Velasco y Díaz de Rada 2006; Villalobos et al., 2015), poniendo énfasis en la redacción de descripciones densas donde se haga explícita la conexión entre los individuos y sus entornos -en este caso socioeconómicos, institucionales y lingüísticos- para así revelar características constitutivas de su cultura (Geertz, 2003; Goetz y LeCompte, 1988; Taylor y Bogdan, 2000). Existe por parte de las investigadoras -en tanto personas oyentes- una constante revisión teórica y práctica de los modos a través de los cuales es posible explorar, interpretar, describir y reproducir los contenidos de una cultura de la que no forman parte. Las entrevistas individuales semiestructuradas con los/as estudiantes se realizaron en LSch, en una triada participativa entre entrevistadora, estudiante e intérprete, abarcando diferentes aspectos cotidianos de sus experiencias en la escuela regular. Reconocer la presencia de capitales lingüísticos diferentes, donde oyentes han sido privilegiados por sobre no oyentes dentro de una organización social en que impera la escucha fue un foco de atención para la aplicación tanto de las observaciones como de las entrevistas, prestando atención a la corporalidad en conjunto de los/as estudiantes (señas, miradas, gestos, intenciones comunicativas manifestadas mediante movimientos faciales o con extremidades, etc.) (Taylor y Bogdan, 2000).

Las entrevistas con profesores/as de educación especial, intérpretes de LSch y personal directivo se complementaron con grupos de discusión en los que participaron duplas docentes e intérpretes de señas. Estas estrategias fueron basadas en un diálogo más bien abierto e informal, sin embargo, también se abordaron preguntas específicas orientadas a llenar los posibles vacíos en relación a nuestras comprensiones iniciales sobre los/as

\footnotetext{
4 "Estudio cualitativo sobre entendimientos y prácticas en torno a los Programas de Integración Escolar y la Educación Especial en Chile”.
} 
estudiantes sordos/as y su cultura, tanto dentro de las aulas como en sus respectivos hogares (Velasco y Díaz de Rada, 2006).

El material transcrito y las notas de campo fueron clasificados mediante codificación abierta y axial, priorizando el rescate de significados y discursos (Charmaz, 2007; Flores y Naranjo, 2013; Strauss y Corbin, 2002; Taylor y Bogdan, 2000). Así fue posible reconocer elementos simbólicos y materiales de producción y co-construcción de discursos y prácticas educativas, los cuales fueron procesados mediante una reflexión selectiva, además de reducidos y organizados de modo situado y triangulado, para dar cuenta no sólo de los intereses investigativos de las observantes, sino que también de las dinámicas cotidianas de cada escuela (Álvarez, 2011). El análisis de los datos derivados implicó conectar la experiencia de los/as estudiantes sordos/as con vivencias escolares y lingüísticas que generalmente encontramos asociadas a la oralidad y la rehabilitación (Herrera y de la Paz, 2019). Por ello, optamos por un giro hacia la representación de subjetividades más allá de la comparativa con las capacidades de estudiantes oyentes; más bien, destacamos los relatos contextualizados en saberes y experiencias del aprendizaje y la enseñanza, elementos indisociables en la comprensión de contenidos co-creados entre estudiantes y sus intérpretes, al momento de comunicarse con profesores/as y compañeros/as. Así, los hallazgos que presentamos ilustran prácticas de aula desde la perspectiva de la observación externa por parte de las investigadoras, como también desde la perspectiva situada de estudiantes sordos/as y sus docentes.

\section{Resultados}

\subsection{Significación de la lengua de señas}

Julieta asiste a 1ero medio en el Colegio Andes, un colegio municipal de la zona sur de Santiago. Hasta tercero básico asistió a una escuela especial, con modelo socioantropológico donde aprendió LSch. Luego se integró al Colegio Andes, donde trabaja su madre. Recibió un implante coclear luego de haberse cambiado de colegio, pero continúa comunicándose por LSch. En las conversaciones que sostuvimos con Julieta no abordamos el tema de su operación, pero su intérprete atribuye la persistencia de Julieta a comunicarse a través de LSch a que la operación no fue exitosa. Sin embargo, la literatura abre interesantes preguntas sobre el impacto psicológico que sufren adultos y adolescentes cuando se los empuja a adoptar una forma de comunicación que implica algún nivel de negación de su identidad. Al respecto, Paddy Ladd (2003) afirma que "aunque los Sordos experimentan el mismo porcentaje de desórdenes psiquiátricos "genéticos" que el resto de la población, hay una clara diferencia en los porcentajes de los problemas emocionales y de conducta 'inducidos por la vida"' (Ladd, 2003, p.7). Aparte del intérprete asignado por la escuela, nadie en la familia de Julieta ni en su curso tiene un manejo adecuado de LSch, por lo que ella no tiene muchas opciones de comunicación.

Daniel, quien asiste a 7 mo básico en el mismo colegio que Julieta, también estuvo en una escuela especial para sordos antes de entrar al establecimiento, y a pesar de que su escuela seguía un modelo socio-antropológico, no desarrolló un manejo acabado de la LSch. Daniel, si bien no tiene implante posee un remanente auditivo, por lo que utiliza un audífono. Sin embargo, cuenta que le molesta y por eso lo apaga. Dentro de los objetivos de la profesora de educación especial que lo apoya se encuentra la mejora de la LSch, aunque ha priorizado la adaptación socio-escolar y Daniel aún no maneja LSch con precisión. 
En los casos de Daniel y Julieta, la LSch por sí sola no es vehículo para la inclusión pues Julieta sólo se comunica con su intérprete, y Daniel basa su comunicación principalmente en gestos. Pese a manejar una lengua, la situación de Julieta no es mejor que la de Daniel, pues no le facilita el aprendizaje ni el desarrollo de habilidades sociales. Vemos que si la LSch no se incorpora a toda la comunidad educativa y se entiende como una manera usual de comunicación entre estudiantes oyentes y sordos, no es más que un traductor de contenidos. La necesidad de que las escuelas regulares a las que asisten los estudiantes sordos sean bilingües queda en evidencia como se observa en los casos de Daniel y Julieta. Sin embargo, tal objetivo no se encuentra en el plan de desarrollo institucional de su colegio. En el siguiente fragmento la profesora de educación especial de Julieta relata cómo el escaso conocimiento de LSch dentro de la comunidad escolar crea barreras entre estudiantes sordos/as y sus profesoras/es y compañeros/as, considerando que estos últimos no se interesan ni interactúan con sordos/as y que los/as intérpretes actúan como traductores:

En el otro colegio donde trabajé también me pasaba lo mismo, también la intérprete hacía muchas cosas con la niña y la sociabilización se enfrascaba sólo en lo que conversaba con ella, o con los mismos sordos que estaban ahi en el colegio; y los problemas que tenía eran con los mismos sordos, es como todo... en comunidad, actuaban así. (Entrevista a Mónica, Profesora de Educación Especial Colegio Andes)

La situación ilustrada contrasta con la experiencia de Daniel, quien, aunque no entiende los contenidos de la misma forma que sus compañeros/as oyentes y requiere del vínculo lingüístico que ha creado con su intérprete, realiza acciones para participar de la clase no sólo en términos de aprendizaje de contenidos sino que, además, en aspectos de interacción social:

\begin{abstract}
La única que habla con Daniel es Clara [intérprete de Daniel] y la interpretación de la clase se la hace sólo a él, no a todo el curso. Los estudiantes de la sala no están con Daniel, nadie le habla ni interactúa con él, pero él se ve contento conversando con Clara. Ella le traduce algunas cosas que él pregunta sobre la clase. La actitud de Daniel, pese a la indiferencia de sus compañeros, es hacia el resto: está abierto hacia lo que tiene a su alrededor, sigue con su mirada los movimientos de la profesora y de sus compañeros, se ríe y hace gestos a los demás. (Notas de campo clase de Lenguaje, Colegio Andes)
\end{abstract}

Sin duda la situación de Daniel es problemática, pero muestra cómo al no haber una significación compartida sobre la LSch en la escuela, él debe abrir su comunicación con los demás por otras vías, sin encapsularse en la clase junto a su intérprete. La apertura de Daniel hacia los demás lo caracteriza, y es muy diferente a Julieta quien es retraída y no se conecta con sus compañeros de clase, presentando algunos de los rasgos psicológicos, emocionales y conductuales que indica la literatura citada.

\title{
4.2. Roles educativos de los/as intérpretes de LSch
}

Aunque los PIE determinan los criterios de ingreso a escuelas regulares, no especifican la forma en que se debe enseñar a los/as estudiantes sordos/as dentro de las salas de clase, por lo que la manera en que los/as intérpretes se integran al proceso de docencia no está reglamentada por lo tanto, sus prácticas son diversas. La presencia de un intérprete supone que los/as estudiantes sordos/as manejen LSch, situación que no siempre sucede ya que su enseñanza no está contemplada para el aula regular, y cuando sucede, ocurre en el aula de recursos, en los tiempos en que docentes de educación especial trabajan de manera individual con los/as estudiantes. La planificación de clases que hacen docentes 
regulares y de educación especial no incluye a los/as intérpretes de LSch, y por la forma en que realizan su interpretación (replicando la clase únicamente para el/la sordo/a), suceden situaciones en las que se adaptan como profesores/as o compañeros/as de clase, desarrollando roles que desbordan su cometido:

Julieta está sentada en la primera fila de su sala. Conversa con su intérprete y con
Mabel, la profesora de educación especial de su curso. Puede seguir bastante bien el
ritmo de la clase ya que Clemente no sólo le traduce, sino que también le explica los
contenidos. Cuando él no entiende algo (matemáticas de Primero Medio puede ser
difícil), él -no Julieta- se acerca a Sonia, la profesora regular, y le pregunta para
luego ir a explicarlo a Julieta. (Observación Matemáticas 1 medio, Colegio
Andes)

De esta forma, Julieta queda desprovista de su profesora de Matemáticas, quien se supone es especialista en esta pedagogía, y también se encapsula con su intérprete en vez de participar de la misma didáctica que sus compañeros/as. Al preguntarle a Clara, intérprete de Daniel, cómo imagina un aula donde se propicie la inclusión del lenguaje para todos y el aprendizaje de personas sordas en particular, nos comparte lo siguiente:

\section{Lo ideal sería que yo esté adelante y mantener la distancia como con cualquier alumno, pero ¿̇qé pasa?, que se pierde mucho la materia, ¿̇por qué? Porque, por ejemplo, las clases están hechas para que los niños copien de la pizarra, y el profesor habla mientras los niños están copiando ¿̇y el sordo? (Entrevista a Clara, intérprete LSch Colegio Andes)}

Proponemos que una posibilidad de funcionamiento alternativo a este modelo es que la LSch se incluya en el currículo de las escuelas con estudiantes sordos/as y que el/la intérprete traduzca los contenidos al lado del/a profesor/a. Así el/la estudiante con DA podría hablar directamente con el/la profesor/a o con sus compañeros/as, valiéndose del/a intérprete y la LSch como herramientas presentes para todos/as en la escuela. Esto implicaría cambiar dinámicas pedagógicas y didácticas que están pensadas para una comunidad oyente.

\subsection{Tensiones respecto a la integración de estudiantes sordos}

Margarita asiste a cuarto básico en el Colegio Buen Pastor, un colegio municipal de la zona sur de Santiago. Asistió hasta tercero básico a una escuela especial, de modelo sociocultural, donde aprendió LSch. Luego se integró al Colegio Buen Pastor, donde cuenta con el apoyo de una psicopedagoga y una profesora de educación especial. Ninguna de las dos tiene un manejo acabado de LSch, en cambio Margarita la maneja bastante bien.

Para las/os docentes regulares enfrentarse a la integración de estudiantes sordos/as en su aula sin contar con un/a intérprete supone un desafío. De ahí que algunos/as profesores/as critiquen el modelo de inclusión a toda costa y su exigencia a desempeñar un rol que les incomoda, principalmente, porque no se ajusta a sus prioridades pedagógicas:

Para mí, como profesora básica, para abordar a Margarita tuve muchas controversias con Sara (profesora de educación especial) porque yo no me sentía apta, no tenía las herramientas, no tenía las habilidades para otorgarle a Margarita lo que ella necesitaba. Porque si a mí me dicen que la vamos a incluir, ya, ¿̇pero cómo la vamos a incluir? No es sentarla en una sala y que ella mire lo que yo hago. Entonces para mí fue un desafio gigantesco. Puedo decir que no encuentro que le hayamos dado lo que ella necesitaba. Aunque sí logramos lo que a ella le preocupaba y que frente a la sociedad la disminuía; que era leer y conocer los números, porque ella llegó a 4to básico sin reconocer lo que uno hace de kínder a lero básico: reconocer vocales, letras, visualizar, reconocer sonidos. Después me encuentro con que querían que hiciera la 
prueba SIMCE (Sistema de medición de la calidad de la educación). ¿Cómo una niña que ni siquiera tenía los objetivos mínimos de 4to básico iba a rendir un SIMCE? (Grupo de discusión, Patricia, profesora de Matemáticas, Colegio Buen Pastor)

Los/as profesores/as regulares desarrollan frustraciones que usualmente intentan ser mediadas a través de las profesoras de educación especial o intérpretes de LSch:

\begin{abstract}
Primero está el tema de que la profesora de educación especial se tiene que poner de acuerdo, o tiene que planificar con la profesora regular, yo ahí sólo tengo conexión con Dora [profesora de educación especial], con la profesora regular no. (Entrevista Clara, intérprete LSch Colegio Andes, julio 2018)
\end{abstract}

Lo anterior muestra un modo en que docentes regulares delegan funciones didácticas y pedagógicas en sus colegas: la profesora regular no establece una relación educativa con sus estudiantes sordos/as pues delega esa interacción en la profesora de educación especial, haciendo invisible la presencia de estudiantes sordos/as. Por otra parte, las profesoras de educación especial no son necesariamente especialistas en DA, como ocurre en los casos de las profesoras de educación especial que apoyan los cursos de Margarita y de Julieta y no manejan LSch, quedando excluidas de las dinámicas que establecen las estudiantes con sus intérpretes. El apoyo que brindan las profesoras de educación especial a los/as sordos/as es más bien en temas generales, no relacionados con superar barreras de comunicación. Además, deben cubrir las demandas de otros/as estudiantes de la clase etiquetados con NEE y al tener más competencias para atender otras condiciones, se abocan a ellas tendiendo a delegar en los/as intérpretes el acompañamiento de estudiantes sordas/os.

\title{
4.4. Aprendizaje de la LSch por parte de la comunidad oyente
}

Ante las situaciones descritas, retomamos que el aprendizaje de LSch por parte de todo el estudiantado es fundamental para la inclusión. Por una parte, es un hecho que no todas/os las/os estudiantes con DA conocen la LSch antes de entrar al colegio, por lo que hace falta que dentro de los PIE se destinen horas especiales a la enseñanza de dicha lengua; y por otra, es necesario que el resto de los/as estudiantes maneje LSch para comunicarse, en especial cuando el/la intérprete no está disponible. Cabe destacar que, aunque exista voluntad por parte de los/as estudiantes oyentes por cambiar el patrón y aprender la LSch, no existen estrategias en esa dirección por parte de los equipos directivos y docentes ni se observan métodos orientados a desarrollar habilidades grupales entre estudiantes que les permitan incluir a sordos/as:

En primero medio, con Julieta, yo creo que el curso no está tan atento. Algunos chicos
saben socializar con ella, pero no están todos en la misma. Algunas chicas, creo que
están desde muy chicas con ella, y ellas son las que saben algunas palabras en señas y
se comunican directamente. Otros han ido aprendiendo, pero será un grupo de 1o a
15 niños, o un poco menos, que sabe algunas palabras de señas y se comunican. Ahora,
a este curso han llegado niños nuevos, entonces, con Clemente [intérprete LSch del
curso de Julieta] el otro día estábamos diciendo "tenemos que hacer algún taller de
señas para el curso, porque como hay chicos nuevos, se ha segregado que sólo el grupo
que sabe señas sea el que se conecta con Julieta. (Entrevista Mónica, Profesora de
Educación Especial Colegio Andes)

La imposibilidad de comunicarse con más personas que el/la intérprete en el colegio posibilita y refuerza la dependencia emocional de los/as estudiantes sordos/as en relación a sus intérpretes u otro/a adulto/a que maneje LSch. Además, plantea dilemas profesionales sobre los límites del trabajo de los/as intérpretes en términos materiales y emocionales: 
Cuando llegué me dijeron "es complicado, porque Julieta ha tenido hartos intérpretes, emocionalmente es súper inestable, entonces necesitamos a alguien que sea fuerte, y en realidad, bien estricta con ella", yo no tenía problema en ser estricta (ríe) asi que funcionó. Estuve el 2015 y 2016 con Julieta y el 2017 cambiamos a medio año, y el otro semestre lo tomaba Clemente, así que yo me quedaba con Daniel. Nos dividimos mitad y mitad porque Julieta estaba siendo muy dependiente de mí. $\Upsilon$ tampoco es bueno, porque yo soy su intérprete y no podemos traspasar ese nivel de confianza. Es cierto, se generan estos otros vínculos, porque uno está todo el día con el estudiante, y al final Julieta... como la familia no conversa con ella en señas, llegaba el día lunes y me contaba todo, pero todo (con énfasis), pasaba por lo menos una hora y la profesora estaba haciendo otra cosa. Después llegaba el fin de semana y me seguía hablando por Whatsapp, entonces ya había que cortar un poquito el cordón ¿̇y cómo lo hacíamos?, cambiando de intérprete. Justo el año anterior había llegado Clemente, porque había llegado Daniel también al colegio. (Clara, intérprete LSch Colegio Andes)

\subsection{Asuntos a destacar sobre el uso de Aulas de Recursos ${ }^{5}$}

En el marco de las posibilidades que entrega la reglamentación para los PIE, el aula de recursos es un espacio frecuentemente usado para la enseñanza individual de estudiantes sordos/as. Esto puede tener consecuencias positivas o negativas, dependiendo de las disposiciones institucionales y pedagógicas tras su uso, principalmente las relacionadas a la conceptualización de la discapacidad y las NEE en la escuela. Llevar a los/as estudiantes al aula de recursos puede potenciar la nivelación de sus aprendizajes previos y reforzar contenidos actuales para que luego puedan integrarse al aula regular. Pero también puede ser una acción segregadora, como por ejemplo cuando se utiliza para sacar del aula a los/as estudiantes del PIE y no interrumpir con su comportamiento el desarrollo de la clase. Aunque las políticas orientadas a la inclusión consideran el aula de recursos como una herramienta complementaria, hay escuelas en que los/as estudiantes del PIE pasan la mayor parte del tiempo en ellas, como es el caso de Margarita en el Colegio Buen Pastor:

Subo a la sala de coordinación PIE (que es también el aula de recursos) donde
Margarita está haciendo una prueba de matemáticas. Ahí Sara, la profesora de
educación especial, me cuenta que Margarita está dos años atrasada en Matemáticas,
Lenguaje y Ciencias Sociales y Naturales, por lo que mayormente trabaja con María,
una psicopedagoga en el aula de recursos. Me doy cuenta entonces que no estará
presente cuando observe las clases regulares en su curso. Según Sara, Margarita se
"perdió en la escuela especial" porque no avanzó curricularmente, sino que sólo en el
desarrollo del lenguaje de señas. (Observación aula de recursos Colegio Buen
Pastor)

Margarita presenta un manejo adecuado de LSch ya que estuvo hasta tercero básico en una escuela para sordos/as. Al ser entrevistada notamos que puede expresarse con claridad, es muy comunicativa y puede darse a conocer, lo que no ha logrado del todo con sus compañeras de clase pues relata haber tenido algunos problemas con ellas. Nos cuenta que se entretiene en el aula de recursos con su profesora de educación especial, aunque también le gusta tener un espacio con sus compañeras de clase. Por un lado, lo que ella valora del aula de recursos es que es un lugar en el que puede comunicarse con fluidez en su lengua. Además, en el aula de recursos puede seguir su propio ritmo de aprendizaje.

${ }^{5}$ Sala con espacio suficiente y funcional para contener la implementación, accesorios y materiales didácticos, u otros, necesarios para satisfacer los requerimientos de apoyo individual o en pequeño grupo de los distintos alumnos NEE. Su existencia es obligatoria para los establecimientos educacionales con PIE, con excepción de aquellos considerados rurales y que cuentan con cursos multigrados (MINEDUC, 2013, p.13). 
Para ella el lenguaje oral es complejo y aunque utiliza un audífono que le otorga un leve margen de audición, e intenta leer los labios cuando se concentra, se confunde y debe recurrir constantemente a su intérprete o psicopedagoga, perdiendo el hilo de la clase. Entonces, le preguntamos qué pasa si María o Sara (sus profesoras) no están junto a ella, y nos cuenta que a pesar de no entender, trata de mantener un cierto orden copiando desde el cuaderno de alguna compañera que tenga sentada cerca. Por otro lado, lo que valora de las clases que comparte con sus compañeras oyentes, sólo Artes y Educación Física, es la posibilidad del juego y así sentirse parte del curso pese a pasar más tiempo en el aula de recursos que en la sala con sus compañeras:

\begin{abstract}
Durante el desarrollo de la clase de educación física, las alumnas forman dos equipos, uno a cada lado de una red de voleibol. Margarita toma a su amiga Daniela de la mano y pasan de un lado a otro. Se supone que tienen que ponerse de acuerdo en las posiciones que cada una tendrá durante el juego, también en el nombre de su equipo. Margarita mira al profesor mientras habla, pero se ve que no entiende lo que dice. María, la psicopedagoga que está 'a cargo' de Margarita y maneja un mínimo de LSch fue a su oficina durante un momento y no ya no está en la clase. El profesor toca el pito y dice que hay que estar 'congeladas' (sin moverse) antes de iniciar. Empieza un juego con pitazos y actividades en que se tienen que coordinar para cambiar puestos, recibir pelotas, aplaudir, etc. Margarita no escuch a el pito, sino que reacciona mirando a sus compañeras. Se la ve contenta en la actividad, pero también se nota cuando está aburrida o cuando tiene cara de no entender cuando las compañeras hablan o el profesor da instrucciones. Si Margarita gana un punto, celebra con su amiga Daniela; cuando es lenta, las otras chicas le aletean y le ayudan a reaccionar con mímica. (Observación clase de Educación Física Colegio Buen Pastor)
\end{abstract}

Para Margarita, tanto el aula regular como el de recursos juegan un rol importante en su experiencia escolar. Por esto consideramos que el aula de recursos debe ser entendida y gestionada de manera contextualizada en las escuelas. Para ello, las políticas educativas deben ofrecer lineamientos claros pero flexibles, que favorezcan la articulación entre factores individuales y factores sociales, culturales e institucionales. El uso permanente del aula de recursos sin combinarlo con permanencia en la sala regular impide la inclusión pedagógica y social de los/as estudiantes sordos/as. Es necesario revisar la forma en que se combinan ambas modalidades para aquellos/as estudiantes que necesitan reforzar sus aprendizajes antes de unirse completamente al aula regular.

\title{
5. Discusión
}

De acuerdo a las experiencias analizadas se puede decir que las/os estudiantes sordas/os que formaron parte de este estudio se encuentran integrados en escuelas regulares, aunque no incluidos. Esto se debe a causas multifactoriales de los casos observados, entre las que destacamos:

- Debilidades de los modelos de administración y financiamiento para la educación especial: existen tensiones en las prioridades escolares respecto a los recursos materiales y humanos disponibles para atender a la especificidad comunicativa de estudiantes sordos/as, ya que las escuelas enfrentan responsabilidades que limitan sus posibilidades de acción para la inclusión, debiendo centrarse en criterios de acreditación y rendición de cuentas para obtener financiamiento estatal, usualmente medidos en pruebas estandarizadas (SIMCE) y planes estratégicos de mejora en el rendimiento, que no necesariamente se enfocan en los recursos necesarios para atender a la diversidad dentro de las aulas. 
- Dificultades en la planificación colaborativa entre docentes e intérpretes: los tiempos de planificación en las escuelas son escasos y poco fiscalizados, muchas veces interpretados como espacios "libres" para cumplir con diversas demandas institucionales, dificultando la debida coordinación entre docentes e intérpretes para planificar la enseñanza y los roles a desempeñar de manera que el/la docente asuma la enseñanza de los/as estudiantes sordos/as y no el/la educador/a diferencial o intérprete, como ha mostrado este estudio. Esto dificulta que las lecciones estén diseñadas universalmente, es decir, orientadas hacia la comprensión e interacción de la diversidad de estudiantes en el aula, teniendo especial consideración con la presentación de los contenidos mediante varias formas de enseñanza aprehensibles por quienes enfrentan mayores barreras para acceder al aprendizaje.

- Enseñanza y evaluaciones poco diferenciadas: lo anterior también provoca deficiencias en las adecuaciones curriculares. Los/as sordos/as indican que les cuesta adaptarse al modo de los/as oyentes, sin embargo, el lenguaje escrito sigue siendo la forma para evaluar sus aprendizajes. Por tanto, se hace necesario incorporar el uso de elementos alternativos de comunicación y evaluación, que además podrían beneficiar el aprendizaje de todo el resto del curso, como por ejemplo presentaciones visuales adaptadas a las condiciones de lectoescritura de los/as estudiantes sordos/as En este sentido, las orientaciones del Decreto 83 que promueve la diversificación de la enseñanza son un insumo para implementar estrategias pedagógicas más específicas para estudiantes con DA.

- Bajo interés de docentes regulares por los procesos de inclusión: preocupa la poca motivación docente por incorporar la LSch a sus clases. Hemos visto que esto tiene que ver, por un lado, con directrices institucionales, pues la distribución de horas no lectivas es limitada y no hay espacio para aprender LSch y planificar clases incorporando de mejor manera la perspectiva bilingüe. Por otro lado, creemos importante mencionar el poco interés que manifiestan estos docentes respecto a herramientas que son vistas como opcionales dentro de sus trabajos diarios, como los talleres de LSch ofrecidos por intérpretes. El rol de los/as adultos/as que conviven con estudiantes sordos/as es fundamental en términos de mediación entre los diversos dominios que componen el aprendizaje: los/as cuidadores familiares, el profesorado y los/as profesionales paradocentes vinculan las esferas donde los/as niños/as se desarrollan, y aunque muchas veces su capacidad de agencia sobre ellas es limitada, pueden entablar conexiones a través de interacciones pedagógicas y comunitarias dentro de la escuela, tomando decisiones que tiendan hacia la inclusión y el beneficio lingüístico e identitario de sus niños/as (Domínguez, 2009; Swanwick, 2015). La falta de interés docente e institucional afecta también la motivación y el interés de los/as estudiantes oyentes por la inclusión.

- Dificultades en la comunicación y en el formato de interpretación en aula: para los/as estudiantes sordos/as no contar con un/a intérprete, profesores/as y compañeros/as que manejen LSch les impide participar de la clase. En algunos casos, los/as estudiantes se ven expuestos a situaciones críticas y frustrantes y en otros, la ausencia de LSch deviene en que realizan sus clases mayoritariamente en el aula de recursos con apoyo de la profesora de educación especial. Además, las dificultades que enfrentan los/as intérpretes de LSch por 
falta de orientaciones respecto a su rol en las aulas regulares y por falta de estrategias efectivas para conectarse con sus colegas docentes puede afectar la trayectoria escolar de estudiantes sordas/os, como señalamos, en aspectos de aprendizaje y participación en clases. Si la interpretación no está integrada a la dinámica de la clase, produce segregación del estudiante sordo/a con su intérprete y levanta una barrera para la inclusión. Finalmente, la díada que se forma entre estudiante e intérprete puede ser conflictiva cuando se produce una relación de dependencia entre estudiante e intérprete, con una sobre exigencia afectiva para este/a último/a. El trabajo docentes regulares y especiales e intérprete debe ser unificado y articularse en torno a objetivos compartidos. La división del trabajo que observamos, en el que el/la docente regular le comunica el contenido de la clase a la profesora de educación especial para que ésta informe a el/la intérprete para que 'se encargue' de enseñar a el/la sordo/a, dificulta el tránsito desde el enfoque de la integración al de una práctica verdaderamente inclusiva.

\section{Conclusión}

Lo que puede ofrecer la política pública en el marco del PIE será siempre limitado, aún cuando existan espacios para promover y perfeccionar escuelas especiales y escuelas regulares de manera que funcionen paralelamente, propiciando interconexiones y herramientas para los oyentes y su interacción en espacios (no solamente escolares) con personas sordas. En ese contexto, surge el dilema entre la inclusión y el derecho de los/as estudiantes a aprender en un ambiente en su lengua. Desde una perspectiva de derechos, el sistema escolar debiera ofrecer a los/as estudiantes sordos/as la oportunidad de acceder a una educación bilingüe de buena calidad, considerando la enseñanza de la LSch no solo a el/la sordo/a y su intérprete, sino también al resto de los miembros de la comunidad escolar. Solo así, los/as estudiantes sordos/as podrán compartir con sus compañeros/as y profesores/as tanto en espacios de orden académico como social, sin que las exigencias se centren solo en la DA. Sin embargo, si para el contexto chileno no es posible asegurar ese derecho en las escuelas regulares, debe existir la posibilidad de elección para aquellos/as estudiantes que prefieran asistir a una escuela especial con enfoque en su lengua. La extensión de las políticas llamadas inclusivas ha puesto en jaque la existencia de las escuelas especiales. Respecto a nuestros hallazgos es importante notar que los/as estudiantes que han adquirido la LSch como primera lengua pueden ver detenido su desarrollo en las escuelas regulares si es que estas no cuentan con un ambiente en el que la comunidad educativa incorpore la LSch. En razón de ello consideramos que cualquier cambio en la oferta de matrícula en escuelas especiales debe ir de la mano con el fortalecimiento de las capacidades de las escuelas regulares de recibir y hacerse cargo del aprendizaje de estudiantes sordos/as y con NEE.

Aunque las narrativas de las/os participantes despliegan variadas interpretaciones sobre el significado de la inclusión, algunas poniendo fuertes limitaciones a las posibilidades reales de ésta, sostenemos que las prácticas docentes son fundamentales para favorecer la participación y el aprendizaje de estudiantes dentro de las salas, mientras que las prácticas de la comunidad escolar (estudiantes, familias, docentes y paradocentes) favorecen la inclusión fuera del aula. Si bien parece necesario realizar diversas modificaciones normativas en pro de la inclusión en tanto concepto aplicado, más allá de lógicas administrativas-gerenciales, afirmamos que para que la multiplicidad de interpretaciones 
sobre inclusión tenga una cabida armónica para los/as diversos/as profesionales de la educación y suficiente para los/as estudiantes, sería importantísimo que las escuelas adopten modelos ecológicos donde el aprendizaje se comprenda como un proceso con múltiples ámbitos de desarrollo (físicos, cognitivos y emocionales), atendiendo a contextos e interacciones sociales, culturales y lingüísticas entre otras. A su vez, esto podría permitir significar territorialmente lo que las escuelas entienden como discapacidad y NEE, superar dilemas entre proveer atención especializada dentro de aulas regulares y equilibrar el peso de factores individuales y sociales a la hora de diseñar clases orientadas a la diversidad cognitiva, cultural, lingüística.

\section{Agradecimientos}

Esta investigación fue financiada por ANID PIA CIE 160007 y desarrollada por el Centro de Justicia Educacional de la Universidad Católica de Chile.

\section{Referencias}

Ainscow, M., Booth, T. y Dyson, A. (2006). Improving schools, developing inclusion. Routledge.

Ainscow, M. (2008). Understanding the development of inclusive schools. Falmer Press.

Álvarez, C. (2008). La etnografía como modelo de investigación en educación. Gazeta de antropología, 24(1), 1-15. https://doi.org/10.30827/Digibug.6998

Álvarez, C. (2011). El interés de la etnografía escolar en la investigación educativa. Estudios Pedagógicos, 37(2), 267-279. http://doi.org/10.4067/S0718-07052011000200016.

Antia, S., Stinson, M. y Gonter-Gaustad, M. (2002). Developing membership in the education of deaf and hard-of-hearing students in inclusive settings. Journal of Deaf Studies and Deaf Education, 7(3), 214-29. https://doi.org/10.1093/deafed/7.3.214

Becerra, C. (2013). Interculturalidad y ciencias de la educación. Boletín Científico Sapiens Research, $3(2), 2-7$.

Becerra, C. (2020). Inclusión e interculturalidad para la cultura sorda: Caminos recorridos y desafíos pendientes. Revista de Investigación Educativa de la REDIECH, 11, 1-23. https://doi.org/10.33010/ierierediech.v1 1 io.792

Bernal-Martínez de Soria, A. (2008). Temas centrales de la antropología de la educación contemporánea. Educación y Educadores, 11(1), 129-144.

Camacho, M. de la C. y Pérez, M. (2014). Aspectos que influyen en las competencias estratégicas didáctico curriculares para atender alumnado sordo desde la voz del profesorado. Profesorado. Revista de Currículum y Formación de Profesorado, 18(1), 341-366.

Canals, C., Aguirre, C., Blanco, C., Fábrega, F., Mena, C. y Paulus, N. (2019). El “voucher” a la chilena. Reflexiones sobre elección escolar y financiamiento educacional. Estudios Pedagógicos, 45(1), 137-150. https://doi.org/10.4067/S0718-07052019000100137

Charmaz, K. (2007). Constructing grounded theory. SAGE Publications Ltd.

Damm, X. y Silva, B. (2017). Políticas públicas chilenas e inclusión social de personas sordas. Perspectiva Educacional, 56(1), 183-201. https://doi.org/10.4151/07189729-Vol.56-Iss.1Art.467

De la Paz, V. y Salamanca, M. (2009). Elementos de la cultura sorda. Una base para el currículum intercultural. REXE. Revista de Estudios y Experiencias en Educación, 8(15), 31-49. 
Domínguez, A. B (2009). Educación para la inclusión de alumnos sordos. Revista Latinoamericana de Inclusión Educativa, 3(1), 45-61.

Echeita, G. y Duk, C. (2008). Inclusión educativa. REICE. Revista Iberoamericana sobre Calidad, Eficacia y Cambio en Educación, 6(2), 1-8.

Fardella, C., Sisto, V., Morales, K., Rivera, G. y Soto, R. (2016). Identidades laborales y ética del trabajo público en tiempos de rendición de cuentas. Psykhe, 25(2), 1-11.

https://doi.org/10.7764/psykhe.25.2.789

Flores, R. y Naranjo, C. (2013). Análisis de datos cualitativos. El caso de grounded theory-teoría fundamentada. En M. Canales (Ed.) Escucha de la escucha: Análisis e interpretación en la investigación cualitativa (pp. 75-114). LOM.

García, R. y López, V. (2019). Políticas de educación especial en Chile (2005-2015): Continuidades y cambios. Revista Brasilera de Educación Especial, 25(1), 1-16. https://doi.org/10.1590/S1413-65382519000100001

Geertz, C. (2003). La interpretación de las culturas. Gedisa.

Giorcelli, L. R. (2004). Making inclusion work. En D. Power y G. Leigh (Eds.). Educating deaf students (pp. 150-160). Gallaudet University Press.

Goetz, J. y LeCompte, M. (1988). Etnografía y diseño cualitativo en investigación educativa. Morata.

Herrera, V. y De la Paz V. (2019). Prácticas pedagógicas y transformaciones sociales. Interculturalidad y bilingüismo en la educación de Sordos. Revista Latinoamericana de Educación Inclusiva, 13(1), 73-88. https://doi.org/10.4067/S0718-73782019000100073

Holz, M. (2018). Datos de la modalidad de educación especial en Chile. Biblioteca del Congreso Nacional.

Huaiquinao, G. y Rebolledo, S. (2019). Una revisión a la experiencia educacional sorda en Chile desde las ciencias del lenguaje. Contextos: Estudios de Humanidades y Ciencias Sociales, 42, 114.

Knoors, H. y Marschark, M. (2014). Learning and context. En H. Knoors y M. Marschark (Eds.) Teaching deaf learners: Psychological and developmental foundations (pp. 217-234). Oxford University Press.

Ladd, P. (2003). Understanding deaf culture. Multicultural Matters.

Lissi, M., Svartholm, K. y González, M. (2012). El enfoque bilingüe en la educación de sordos: sus implicancias para la enseñanza y el aprendizaje de la lengua escrita. Estudios Pedagógicos, 38(2), 299-320. https://doi.org/10.4067/S0718-07052012000200019

Ministerio de Educación. (2007). Necesidades educativas especiales asociadas a discapacidad auditiva: guía de apoyo técnico-pedagógico: necesidades educativas especiales en el nivel de educación parvularia. Unidad de Educación Especial, Ministerio de Educación.

Ministerio de Educación. (2013). Orientaciones técnicas para programas de integración escolar. Ministerio de Educación.

Morales, G. y Morales, V. (2018). Diversidad Sorda: educación y sensibilidad intercultural en una escuela especial de Santiago de Chile. Psicogente, 21(40), 458-475.

https://doi.org/10.17081/psico.21.40.3083

Morales, G. (2019). Percepciones sobre la lengua de señas chilena en la educación de estudiantes Sordos: docente y codocente como sujetos históricos situados comunicativamente en el aula. Revista educación, 43(2), 1-34. https://doi.org/10.15517/revedu.v43i2.31169 
Palacios, R., Larrazabal, S. y Berwart, R. (2019). Educational policies and professional identities: the case of Chilean special educational needs (SEN) teachers under new regulations for SEN student inclusion in mainstream schools. Ethnography and Education, 15(4), 479-492. https://doi.org/10.1080/17457823.2019.1700385

Powers, S. (2002). From concepts to practice in deaf education: A United Kingdom perspective on inclusion. Journal of deaf studies and deaf education, 7(3), 230-243. https://doi.org/10.1093/deafed/7.3.230

Ruiz, N. (2016). El niño sordo en el aula ordinaria. Revista internacional de apoyo a la inclusión, Logopedia, Sociedad y Multiculturalidad, 2(1), 19-32.

Santa Cruz, C. (2019). Es la inclusión educativa siempre justa? El caso de estudiantes chilenos con discapacidades severas. En J.de D. Oyarzún (Dir.), Justicia Educacional en Chile: Más allá de la calidad y la equidad. Latin American Sociology Association (LASA).

Sen, A. (1992). Inequality reexamined. Clarendon Press.

Sisto, V. (2019). Inclusión “a la chilena”: La inclusión escolar en un contexto de políticas neoliberales avanzadas. Archivos Analíticos de Políticas Educativas, 27(23), art. 4. https://doi.org/10.14507/epaa.27.3044

Strauss, A. y Corbin, J. (2002). Bases de la investigación cualitativa. Técnicas y procedimientos para desarrollar la teoría fundamentada. Editorial Universidad de Antioquía.

Swanwick, R. (2015). Re-envisioning learning and teaching in deaf education. En H. Knoors y M. Marschark (Eds.), Educating deaflearners: Creating a global evidence base (pp. 596-614). Oxford University Press.

Taylor, S. y Bogdan, R. (2000). Introducción a los métodos cualitativos de investigación. Paidós.

Terzi, L. (2005). Beyond the dilemma of difference: the capability approach to disability and special educational needs. Journal of Philosophy of Education, 39(3), 443-459. https://doi.org/10.1111/j.1467-9752.2005.00447.x

Valles, B. (2003). Una propuesta para el desarrollo de una política pública para la atención integral temprana de las deficiencias auditivas en Venezuela. Laurus, 9(15), 90-110.

Velasco, H. y Díaz de Rada, A. (2006). La lógica de la investigación etnográfica. Un modelo de trabajo para etnógrafos de escuela. Trotta.

Vesga, L. del S. y Vesga, J. del M. (2015). Una exclusión que se perpetúa: Tensiones entre docentes, niños sordos y niños oyentes en escenarios escolares de Popayán. Revista virtual Universidad Católica del Norte, 46,115-128.

Villalobos, C., Rojas, C. y Torrealba, D. (2015) Gestión de la heterogeneidad en las aulas chilenas. Técnicas, patrones de agrupamiento y sus efectos en el sistema educativo: Un estudio de caso. Revista Latinoamericana de Educación Inclusiva, 9(1), 21-42.

Villalobos, C. y Quaresma, M. L. (2015). Sistema escolar chileno: Características y consecuencias de un modelo orientado al mercado. Convergencia, 22(69), 63-84.

Warnock, M. (2005). Special educational needs: A new look. Philosophy of Education Society of Great Britain Publications.

\section{Breve CV de las autoras}

\section{Sofia Larrazabal}


Investigadora asistente en el Centro de Justicia Educacional (CJE) de la Pontificia Universidad Católica de Chile. Licenciada en Ciencia Política y Relaciones Internacionales por la Universidad Alberto Hurtado y diplomada en métodos cualitativos para la investigación social por la Universidad Diego Portales. Actualmente se desempeña en el equipo de la línea de investigación relativa a inclusión de la discapacidad, donde sus temas de investigación han sido políticas públicas e inclusión, creatividad para la inclusión y formación docente de profesores diferenciales. Sus intereses refieren a relaciones entre sociedad, cultura e instituciones políticas, con especial énfasis en perspectivas multiculturales y feministas. Su trabajo ha sido publicado en Ethnography and Education, Latin American Policy, Sociedade e Cultura y en la revista chilena de antropología Chungará. ORCID ID: https://orcid.org/0000-0001-5868-1820. Email: sofia.larrazabal@gmail.com

\section{Rosario Palacios}

Investigadora Asociada en el Centro de Justicia Educacional (CJE) de la Pontificia Universidad Católica de Chile. Doctora en Sociología por la London School of Economics and Political Science, y Magíster en Planificación Urbana por Columbia University, New York. Actualmente se desempeña en el equipo de la línea de investigación relativa a inclusión de la discapacidad, desarrollando estudios etnográficos y cualitativos que pretenden otorgar una mirada multidisciplinar al tema de la educación. En esa línea se ha centrado en análisis de políticas públicas para la inclusión, creatividad en el aula, formación docente de profesores diferenciales e identidades profesionales, y contextos geográficos de la escuela. Su trabajo ha sido publicado en Ethnography and Education, Latin American Policy, Perspectivas Latinoamericanas, Ethnography, Space and Culture, entre otras. ORCID ID: https://orcid.org/OO00-0001-8839-3520. Email: r.palacios@uc.cl

\section{Victoria Espinoza}

Directora de Investigación del Centro de Desarrollo de Tecnologías de Inclusión y colaboradora en la linea de Investigación y Discapacidad del Centro de Justicia Educacional. Profesora de Educación Básica especialista en Dificultades de Aprendizaje e Inclusión Educativa, Magíster en Psicología, mención en Psicología Educacional, Doctora en Psicología de la Pontificia Universidad Católica de Chile. Sus temas de investigación refieren al proceso de adquisición de la lectura, la educación inicial, el desarrollo de las funciones ejecutivas, la inclusión educativa de estudiantes con discapacidad y las brechas de aprendizaje terminadas por el nivel socioeconómico y su impacto en las posteriores trayectorias de aprendizaje. ORCID ID: https://orcid.org/0000-0001-9462-1139. Email: victoriaespinoza@uc.cl 